\title{
Investigation of sitting postural angle and muscle activity on ageing women workforce during computer task
}

\author{
Nabilla Sofia Mohd Kamil, a , Siti Zawiah Md Dawal ${ }^{2, b}$ \\ ${ }^{1,2}$ Center for Product Design and Manufacturing (CPDM), Faculty of Engineering, University of \\ Malaya, 50603 Kuala Lumpur, Malaysia \\ asofia_kamil@yahoo.com.my, bsitizawiahmd@um.edu.my
}

Keywords: Sitting posture, muscle activity, computer task

\begin{abstract}
Few studies have investigated sitting posture and muscle activity during computer work. This study aims to determine sitting postural angle and its influence on neck and low back muscle activity in ageing women workforce during computer task. Seventeen female workers aged 50 and above participated. Surface electromyography activity was recorded from cervical erector spinae, lumbar multifidus, longgisimus and iliocostalis in 20 minutes computer task. Concurrently inclinometer recordings from upper trunk and pelvis were used to determine back posture. The results for the upper trunk angle show that the posture deviates from the sagittal plane in range -10 to 10 degree during the task. While the pelvic rotated forward and backward in range -15 to 15 degree.Neutral sitting posture with slight extension or flexion of the upper trunk and pelvic posture performed by ageing women workforce during computer work cause reduction in muscle activity. Higher level of muscle activity was indicated in back flexion rather than back extension.
\end{abstract}

\section{Introduction}

The effective role of computers in employment, mainly in clerical and administration sector has resulted many workers to spend time sitting for several hours [1] . A survey conducted by Cheng PL, Dumas GA [2] highlighted sitting as the most common problematic task at work due to factors of uncomfortable posture, fatigue and excessive time in the same posture. A few studies have signified poor sitting posture during computer task is an indicator in the development of low back pain [3, 4]. National Health Interview Survey [5] and National Health and Nutrition Examination Survey Data (1999-2004) reported that back represent the greater rate of pain compared to hips, knee, legs, shoulder, and the upper limb. The survey also stated that back pain is in the highest percentage within ageing population. Stein et.al (1999) defined ageing as the process by which organisms proceed through a physical deterioration of the body. Based on the studies on the bonemuscle relationship in women, age-related bone loss is greatly accentuated by the rapid decrease of estrogen levels occurring as a result of the menopause [6]. Menopause which is an obvious sign of ageing was experience by most women around the age of 50 [7]. A report on 'Women, Ageing and Health' prepared by WHO [8] also described ageing as women age 50 and older. As people aged, the bones lose calcium which cause the trunk becomes shorter while the spinal column becomes curved and compressed [9].

Changes in posture is a serious issue among clinician because postural deviation can produce excessive stress on the musculoskeletal system [10]. Posture of ageing workers during sitting at computer workstation is relatively little studied [11]. Nevertheless, the concepts of ideal sitting posture have a long history in literature [12]. Electromyography and postural angle studies are recognized indicator to determine an optimum sitting posture since different type of sitting posture is prove to cause different effect on muscle activity [13]. In earlier research, Dolan et al (1988) and Anderson (1987) found that erect sitting posture with forward inclination of the upper body usually causes the highest level of muscle activity. In contradiction with erect posture, slump sitting is found to cause the muscle activity decrease and strain on passive structure increase [14]. Several 
studies considering factors of chair, indicated that load on lumbar muscles varies with angle of backrest inclination. However, the posture for a seated person depend not only the design of the chair but also on individuals sitting habits and the nature of the task to be performed.

The main aim of this study was to investigate sitting posture of ageing women workforce during computer task and the effect on muscle activity. Postural angle of upper trunk and pelvic were recorded during the computer task. Simultaneously, neck and low back muscle activity were measured to determine the effect of different postural angle on muscle activity. The study focused on ageing population since the number of this ageing population keeps increasing over time, the participation of this population in the economic development will become a vital issue[15].

\section{Methodology}

Participants

Seventeen subjects consisted of females (age, mean \pm SD: $55 \pm 3.22$ years, range 50-61) aged between 50 to 61 years old were recruited to participate in the experiment. They were staff from various departments in university which work duties mainly involved seated computer work more than 4 hours per day. All participants were in good health and have no problem with normal mobility.

\section{Procedure}

The subjects had to perform 20 minutes computer task without break. The tasks comprised of 10 minutes typing a text from an article followed by another 10 minutes of exercises involving both the mouse and keyboard. These tasks are similar to the standardized job applied by Delisle et. al (2006). The experiment was implemented in a laboratory set up to avoid noise that may disturb the signal of electromyography. The same table and office chair were used for all sessions. Its height and the angle of backrest were also adjustable to accommodate the participants' anthropometric. A standard wired keyboard was used during the typing task and a wired, optical mouse with scroll wheel was used for mouse work. At the start of each experiment session, maximal voluntary contraction (MVC) test for eractor spinae and multifidii were performed. In this study the subjects were instructed to execute back extension in prone laying position. This is because all back muscles were facilitated within a muscle chains and MVCs for the erector spinae can be found during the task.

\section{Data collection}

Postural angle of the upper trunk and pelvis were recorded by Inline 2D inclinometer (NORAXON EMG \& Sensor System). The size of the inclinometer were 3.05 (width) x 3.05 (height) x $3.05 \mathrm{~cm}$ (depth) and the weight was $45.5 \mathrm{~g}$. Inclinometers for measuring upper trunk and pelvic posture were placed at T2 level and sacrum respectively. The inclinometers were attached by surgical tape and positioned to nominally record movements in the sagittal plane. EMG signal were recorded from four muscles: cervical erector spinae, longissimus, iliocostalis and multifidus. These muscles are significant muscle related to back pain. The electrode positions and the procedure to identify the muscle were according to surface EMG for noninvasive muscle assessment (SENIAM) [www.seniam.org]. 


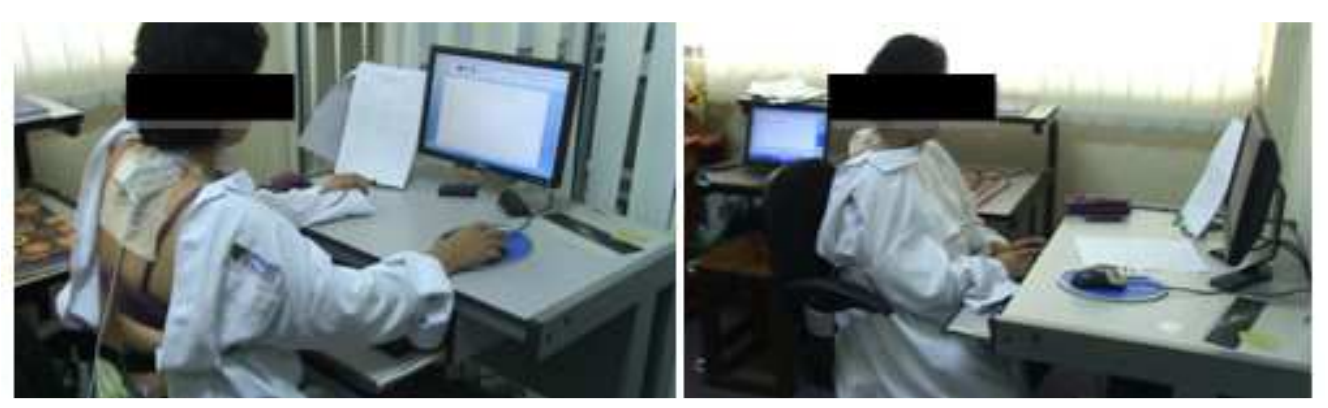

Figure 1. Subject performed 20 minutes computer work

Data analysis

The raw data of EMG were normalized to the maximum voluntary contraction (MVC) to calibrate the microvolt value of the RMS to the percentage of maximum interventions capacity (\%MVC). Muscle activity in this study is reported as a percentage of MVC. Mean percentage of MVC and postural angle of 20 minutes computer work were calculated.

\section{Results}

The postural angle of upper trunk and EMG results of each muscle: cervical erector spinae, longissimus, iliocostalis, multifidus are summarized in Table 1.

Table 1. Upper trunk angle vs. \% MVC

\begin{tabular}{|c|c|c|c|c|c|}
\hline \multirow{3}{*}{\multicolumn{2}{|c|}{$\begin{array}{l}\text { Upper trunk angle, } \alpha \\
\left({ }^{\circ}\right)\end{array}$}} & \multicolumn{4}{|c|}{$\% \mathrm{MVC}$ of EMG } \\
\hline & & \multicolumn{4}{|c|}{ Muscle } \\
\hline & & $\begin{array}{c}\text { Cervical erector } \\
\text { spinae }\end{array}$ & Longissimus & Iliocostalis & Multifidus \\
\hline \multirow[t]{2}{*}{ Backward } & $-10<\alpha \leq-5$ & 11.861 & 8.598 & 8.775 & 4.891 \\
\hline & $-5<\alpha \leq 0$ & 8.884 & 4.060 & 11.533 & 5.283 \\
\hline \multirow{2}{*}{ Forward } & $0<\alpha \leq 5$ & 13.333 & 9.715 & 6.031 & 10.282 \\
\hline & $5<\alpha \leq 10$ & 10.482 & 11.405 & 11.832 & 12.806 \\
\hline
\end{tabular}

The results for the upper trunk angle show that the posture deviates from the sagittal plane in range -10 to 10 degree during the task. For cervical erector spinae muscle, the lowest muscle activity is achieved when the upper trunk angle incline backward in range 0 to -5 degree. The same situation occurred in longissimus muscle. Contrast with ilicostalis muscle, the percentage of MVC is the lowest during forward inclination of the upper trunk. When the upper trunk performed backward inclination in range -5 to -10 degree, multifidus show the lowest muscle activity. The highest percentage of MVC on longissimus, iliocostalis and multifidus are found during forward inclination of group range 5 to 10 degree. As general, the highest percentage of MVC is obtained when the upper trunk is in flexion at range 5 to 10 degree.

The postural angle of pelvic and percentage of MVC results of each muscle: cervical erector spinae, longissimus, iliocostalis, multifidus are illustrated in Table 2. 
Table 2. Pelvic angle vs. \% MVC on computer task

\begin{tabular}{|c|c|c|c|c|c|}
\hline \multicolumn{2}{|c|}{ Pelvic angle, $\beta\left(^{\circ}\right)$} & \multicolumn{4}{|c|}{$\% \mathrm{MVC}$} \\
\hline & & \multicolumn{4}{|c|}{ Muscle } \\
\hline & & $\begin{array}{c}\text { Cervical } \\
\text { erector } \\
\text { spinae }\end{array}$ & Longissimus & Iliocostalis & Multifidus \\
\hline \multirow[t]{3}{*}{ Backward } & $-15<\beta \leq-10$ & 12.819 & 11.424 & 12.370 & 4.581 \\
\hline & $-10<\beta \leq-5$ & 25.461 & 5.666 & 9.523 & 4.646 \\
\hline & $-5<\beta \leq 0$ & - & 9.770 & 4.752 & 4.700 \\
\hline \multirow[t]{3}{*}{ Forward } & $0<\beta \leq 5$ & 14.031 & 5.440 & 5.020 & 10.265 \\
\hline & $5<\beta \leq 10$ & 29.936 & 17.948 & 12.170 & 6.192 \\
\hline & $10<\beta \leq 15$ & 20.238 & 6.664 & 8.060 & 13.976 \\
\hline
\end{tabular}

The results for the pelvic angle show that the posture deviates from the sagittal plane in range -15 to 15 degree during the task. The results in Table 3 indicates that the percentage of MVC for both cervical erector spinae and longissimus were the lowest while the pelvic performed forward inclination at angle range 0 to 5 degree. For iliocostalis and multifidus, the percentage of MVC was the lowest during backward inclination of the pelvic with group range -5 to 0 degree and -10 to -15 degree respectively. Both cervical erector spinae and longgisimus showed the highest percentage of MVC during pelvic in flexion at angle range 5 to 10 degree.

\section{Discussion}

The main findings of this study are sitting postural angle of ageing women workforce and the effect on muscle activity. According to the result, the subjects showed various postural angles within rang -10 to 10 degree for the upper trunk and -15 to 15 degree for the pelvic. The positive value indicates flexion and negative value refer to extension. For the ease of comparison, the data of postural angle was grouping into interval of 5.The result obtained are not much different from the study done by Dumas, Upjohn [1]that identified the deviation of trunk posture of women during computer work was approximately -2 to 10 degree. The difference with result of the current study may be related to the age of the subjects. In Dumas studies, the subjects assessed were younger female workers with mean aged 31.8 years. However, the postural result from this study contradict with a research investigate sitting posture conducted by Mork and Westgaard [11] that reported the trunk flexion is in angle range 15 to 25 degree. The postural data collected during 20 minutes task cannot explained this result, which may be related to long period of work in a sitting posture. In the same study the range of postural angle for pelvic is slightly different compared to this finding which the pelvic rotated backward in range -10 to -20 degree. This is due to the facts that the subjects adopted a flexed posture when seated with backward rotated pelvis and forward rotated upper trunk. It can be seen that the greater muscle activity achieved as the upper trunk inclined forward. According to the findings by Caneiro, O'Sullivan [16] ,increased trunk flexion associated with slump sitting caused greater muscle activation of the cervical erector spinae. The current study also showed the similar trend as the highest percentage of MVC for all muscles is achieved when the upper trunk inclined forward. Apparently the cervical erector spinae need to increase their activity in order to support the weight of the head/neck in this forward head posture. Previous studies report that this type of sitting also results in increased upper cervical spine extension and lower cervical spine flexion (Black et al., 1996). This combined with increased head/neck flexion and neck extensor muscle activity may create focus stress regions in the cervical spine resulting in postural pain. In general, most of the muscles except for iliocostalis present the lowest muscle activity in the upper trunk extension. There are a number of possible causes for these findings. As reported by O'Sullivan et al. (2006), the thoracic upright posture which is achieved via thoracic spinal extensors was described as a substitution pattern where the activation of the thoracic erector spinae muscle resulted in a 
relaxation of the multifidus muscle. The same phenomenon may be the case in the cervical spine where the activation of the long cervico-thoracic extensors results in relaxation of the local cervical spine extensors. In pelvic posture investigation, the lowest muscle activity except for multifidus is produced when the minimal deviation of the pelvic angle from the neutral position is achieved neglected the direction of the inclination. However, the higher muscle activity is associated with forward rotated pelvis. This is consistent with previous studies that indicate active use of these muscles to maintain an upright sitting posture (Mork et.al, 2009). In multifidus muscle, the reduction in sEMG activity with backward rotation of the pelvis, indicative slump sitting, is a kin to the flexion-relaxation phenomenon, however active use of backrest may also contribute. O'Sullivan has been proposed that an optimal sitting posture for low back pain subjects who is more sensitised to flexion or extension is a more neutral position involving slight lumbar lordosis and a relaxed thorax. The same case may be applicable to this study since a reduction of muscle activity was achieved as the upper trunk and pelvic produce a little flexion and extension from the neutral posture.

\section{Conclusion}

In this study it was found that neutral sitting posture with less forward or backward inclination of back posture performed by ageing women workforce during computer work caused reduction in muscle activity. Higher level of muscle activity was indicated in back flexion rather than back extension.

\section{References}

[1] Dumas, G.A., et al., Posture and muscle activity of pregnant women during computer work and effect of an ergonomic desk board attachment. International Journal of Industrial Ergonomics, 2009. 39(2): p. 313-325.

[2] Cheng PL, et al., Analysis of self-reported problematic tasks for pregnant women. Ergonomics, 2006. 49(3): p. 282-292.

[3] Fogleman, M. and R.J. Lewis, Factors associated with self-reported musculoskeletal discomfort in video display terminal (VDT) users. International Journal of Industrial Ergonomics, 2002. 29(6): p. 311-318.

[4] Szeto, G.P.Y., L.M. Straker, and P.B. O'Sullivan, EMG median frequency changes in the neckshoulder stabilizers of symptomatic office workers when challenged by different physical stressors. Journal of Electromyography and Kinesiology, 2005. 15(6): p. 544-555.

[5] National Health Interview Survey, Adult Sample Level File, 2007.

[6] Lang, T.F., The Bone-Muscle Relationship in Men and Women. Journal of Osteoporosis, 2011. 2011.

[7] Gold, E.B., et al., Factors Associated with Age at Natural Menopause in a Multiethnic Sample of Midlife Women. American Journal of Epidemeology, 2000. 159(9).

[8] WHO, Women, Ageing and Health: A Framework for Action, 2007: France.

[9] Information from: http://www.physio-pedia.com/Older_People_Introduction.

[10]Yi-Liang Kuo, et al., Video analysis of sagittal spinal posture in healthy young and older adults. Journal of Manipulative and Physiological Therapeutics, 2009. 32(3).

[11]Mork, P.J. and R.H. Westgaard, Back posture and low back muscle activity in female computer workers: A field study. Clinical Biomechanics, 2009. 24: p. 169-175.

[12]Harrison, D.D., et al., Sitting biomechanics Part I: Review of the Literature. Journal of Manipulative and Physiological Therapeutics, 1999. 22(9): p. 594-609. 
[13]Aaras A, R.H Westgard, and E. Stranden, Postural angle as an indicator of postural load and musculor injury in occupational work station. Ergonomics, 1988. 31(6): p. 915-933.

[14]O'Sullivan, P.B., et al., The effect of different standing and sitting postures on trunk muscle activity in a pain-free population. SPINE 2002. 27(11): p. 1238-1244.

[15]Ahmad, W.I.W., Z. Ismail, and A.H.A. Rahman, Aging and Economic Participation of Older Population in Rural Malaysia. International Journal of Humanities and Social Science, 2011. 1(9).

[16]Caneiro, J.P., et al., The influence of different sitting postures on head/neck posture and muscle activity. Manual Therapy, 2010. 15 p. 54-60. 